\title{
ON THE $x$-COORDINATES OF PELL EQUATIONS THAT ARE PRODUCTS OF TWO PELL NUMBERS
}

\author{
MAHADI DDAMULIRA AND FLORIAN LUCA
}

\begin{abstract}
Let $\left(P_{m}\right)_{m \geq 0}$ be the sequence of Pell numbers given by $P_{0}=0, P_{1}=1$, and $P_{m+2}=2 P_{m+1}+\bar{P}_{m}$ for all $m \geq 0$. In this paper, for an integer $d \geq 2$ which is square free, we show that there is at most one value of the positive integer $x$ participating in the Pell equation $x^{2}-d y^{2}= \pm 1$, which is a product of two Pell numbers.
\end{abstract}

\section{InTRODUCTION}

Let $\left(P_{m}\right)_{m \geq 0}$ be the sequence of Pell numbers given by $P_{0}=0, P_{1}=1$, and

$$
P_{m+2}=2 P_{m+1}+P_{m}
$$

for all $m \geq 0$. This is sequence $A 000129$ on the On-Line Encyclopedia of Integer Sequences (OEIS) [19]. The first few terms of this sequence are

$$
\left(P_{m}\right)_{m \geq 0}=0,1,2,5,12,29,70,169,408,985,2378,5741,13860,33461, \ldots
$$

Putting $(\alpha, \beta)=(1+\sqrt{2}, 1-\sqrt{2})$ for the roots of the characteristic equation $r^{2}-2 r-1=$ 0 of the Pell sequence, the Binet formula for its general terms is given by

$$
P_{m}=\frac{\alpha^{m}-\beta^{m}}{2 \sqrt{2}}, \quad \text { for all } \quad m \geq 0 .
$$

Furthermore, we can prove by induction that the inequality

$$
\alpha^{m-2} \leq P_{m} \leq \alpha^{m-1}
$$

holds for all $m \geq 1$.

Let $d \geq 2$ be a positive integer which is not a perfect square. It is well known that the Pell equation

$$
x^{2}-d y^{2}= \pm 1
$$

has infinitely many positive integer solutions $(x, y)$. By putting $\left(x_{1}, y_{1}\right)$ for the smallest positive solution, all solutions are of the form $\left(x_{n}, y_{n}\right)$ for some positive integer $n$, where

$$
x_{n}+y_{n} \sqrt{d}=\left(x_{1}+y_{1} \sqrt{d}\right)^{n} \quad \text { for all } n \geq 1 .
$$

Furthermore, the sequence $\left(x_{k}\right)_{k \geq 1}$ is binary recurrent. In fact, the following formula

$$
x_{n}=\frac{\left(x_{1}+y_{1} \sqrt{d}\right)^{n}+\left(x_{1}-y_{1} \sqrt{d}\right)^{n}}{2},
$$

2010 Mathematics Subject Classification. 11B39, 11D45, 11D61, 11J86.

Key words and phrases. Pell equation, Pell numbers, Linear forms in logarithms, Baker's method. 
holds for all positive integers $n$.

Recently, Kafle, et al. [10] considered the Diophantine equation

$$
x_{n}=F_{\ell} F_{m},
$$

where $\left(F_{m}\right)_{m \geq 0}$ is the sequence of Fibonacci numbers given by $F_{0}=0, F_{1}=1$, and $F_{m+2}=F_{m+1}+F_{m}$ for all $m \geq 0$. They proved that equation (5) has at most one solution $n$ in positive integers except for $d=2,3,5$, for which case equation (5) has the solutions $x_{1}=1$ and $x_{2}=3, x_{1}=2$ and $x_{2}=26, x_{1}=2$ and $x_{2}=9$, respectively.

There are many other researchers who have studied related problems involving the intersection sequence $\left(x_{n}\right)_{n \geq 1}$ with linear recurrence sequences of interest. For example, [6, 8, 11, 12, 13, 15, 16, 18].

\section{Main Result}

In [7], together with Luca and Rakotomala we studied a problem involving the intersection of Fibonacci numbers with a product of two Pell numbers, so it is also interesting to study the intersection of the $x$-coordinates of Pell equations with a product of two Pell numbers. In this paper, we study a similar problem to that of Kafle, et al. [10, but with the Pell numbers instead of the Fibonacci numbers. That is, we show that there is at most one value of the positive integer $x$ participating in (3) which is a product of two Pell numbers. This can be interpreted as solving the Diophantine equation

$$
x_{n}=P_{\ell} P_{m} .
$$

Theorem 1. For each square-free integer $d \geq 2$ there is at most one $n$ such that the equation (6) holds.

\section{Preliminary Results}

3.1. Notations and terminology from algebraic number theory. We begin by recalling some basic notions from algebraic number theory.

Let $\eta$ be an algebraic number of degree $d$ with minimal primitive polynomial over the integers

$$
a_{0} x^{d}+a_{1} x^{d-1}+\cdots+a_{d}=a_{0} \prod_{i=1}^{d}\left(x-\eta^{(i)}\right),
$$

where the leading coefficient $a_{0}$ is positive and the $\eta^{(i)}$ 's are the conjugates of $\eta$. Then the logarithmic height of $\eta$ is given by

$$
h(\eta):=\frac{1}{d}\left(\log a_{0}+\sum_{i=1}^{d} \log \left(\max \left\{\left|\eta^{(i)}\right|, 1\right\}\right)\right) .
$$

In particular, if $\eta=p / q$ is a rational number with $\operatorname{gcd}(p, q)=1$ and $q>0$, then $h(\eta)=\log \max \{|p|, q\}$. The following are some of the properties of the logarithmic height function $h(\cdot)$, which will be used in the next sections of this paper without 
reference:

$$
\begin{aligned}
h(\eta \pm \gamma) & \leq h(\eta)+h(\gamma)+\log 2 \\
h\left(\eta \gamma^{ \pm 1}\right) & \leq h(\eta)+h(\gamma) \\
h\left(\eta^{s}\right) & =|s| h(\eta) \quad(s \in \mathbb{Z}) .
\end{aligned}
$$

For the proofs of (7) and further details, we refer the reader to the book of Bombieri and Gubler [3].

3.2. Linear forms in logarithms. In order to prove our main result Theorem 1 , we need to use several times a Baker-type lower bound for a nonzero linear form in logarithms of algebraic numbers. There are many such in the literature like that of Baker and Wüstholz from [2]. We start by recalling the result of Bugeaud, Mignotte, and Siksek ([4], Theorem 9.4, pp. 989), which is a modified version of the result of Matveev [17], which is one of our main tools in this paper.

Theorem 2. Let $\gamma_{1}, \ldots, \gamma_{t}$ be positive real algebraic numbers in a real algebraic number field $\mathbb{K}$ of degree $D, b_{1}, \ldots, b_{t}$ be nonzero integers, and assume that

$$
\Lambda:=\gamma_{1}^{b_{1}} \cdots \gamma_{t}^{b_{t}}-1 \neq 0
$$

Then,

$$
\log |\Lambda|>-1.4 \times 30^{t+3} \times t^{4.5} \times D^{2}(1+\log D)(1+\log B) A_{1} \cdots A_{t}
$$

where

$$
B \geq \max \left\{\left|b_{1}\right|, \ldots,\left|b_{t}\right|\right\}
$$

and

$$
A_{i} \geq \max \left\{D h\left(\gamma_{i}\right),\left|\log \gamma_{i}\right|, 0.16\right\}, \quad \text { for all } i=1, \ldots, t .
$$

When $t=2$ and $\gamma_{1}$ and $\gamma_{2}$ are positive and multiplicatively independent, we can use a result of Laurent, Mignotte, and Nesterenko [14]. Namely, let in this case $B_{1}$ and $B_{2}$ be real numbers larger than 1 such that

$$
\log B_{i} \geq \max \left\{h\left(\gamma_{i}\right), \frac{\left|\log \gamma_{i}\right|}{D}, \frac{1}{D}\right\}, \quad \text { for } \quad i=1,2,
$$

and put

$$
b^{\prime}:=\frac{\left|b_{1}\right|}{D \log B_{2}}+\frac{\left|b_{2}\right|}{D \log B_{1}} .
$$

Put

$$
\Gamma:=b_{1} \log \gamma_{1}+b_{2} \log \gamma_{2} .
$$

We note that $\Gamma \neq 0$ because $\gamma_{1}$ and $\gamma_{2}$ are multiplicatively independent. The following result is Corollary 2 in [14].

Theorem 3. With the above notations, assuming that $\gamma_{1}$ and $\gamma_{2}$ are positive and multiplicatively independent, then

$$
\log |\Gamma|>-24.34 D^{4}\left(\max \left\{\log b^{\prime}+0.14, \frac{21}{D}, \frac{1}{2}\right\}\right)^{2} \log B_{1} \log B_{2} .
$$


Note that with $\Gamma$ given by (9), we have $e^{\Gamma}-1=\Lambda$, where $\Lambda$ is given by $(8)$ in case $t=2$, which explains the connection between Theorem 2 and Theorem 3 .

3.3. Reduction procedure. During the calculations, we get upper bounds on our variables which are too large, thus we need to reduce them. To do so, we use some results from the theory of continued fractions.

For the treatment of linear forms homogeneous in two integer variables, we use the well-known classical result in the theory of Diophantine approximation.

Lemma 1. Let $\tau$ be an irrational number, $\frac{p_{0}}{q_{0}}, \frac{p_{1}}{q_{1}}, \frac{p_{2}}{q_{2}}, \ldots$ be all the convergents of the continued fraction of $\tau$ and $M$ be a positive integer. Let $N$ be a nonnegative integer such that $q_{N}>M$. Then putting $a(M):=\max \left\{a_{i}: i=0,1,2, \ldots, N\right\}$, the inequality

$$
\left|\tau-\frac{r}{s}\right|>\frac{1}{(a(M)+2) s^{2}}
$$

holds for all pairs $(r, s)$ of positive integers with $0<s<M$.

For a nonhomogeneous linear form in two integer variables, we use a slight variation of a result due to Dujella and Pethö (see [9], Lemma 5a). For a real number $X$, we write $\|X\|:=\min \{|X-n|: n \in \mathbb{Z}\}$ for the distance from $X$ to the nearest integer.

Lemma 2. Let $M$ be a positive integer, $\frac{p}{q}$ be a convergent of the continued fraction of the irrational number $\tau$ such that $q>6 M$, and $A, B, \mu$ be some real numbers with $A>0$ and $B>1$. Furthermore, let $\varepsilon:=\|\mu q\|-M\|\tau q\|$. If $\varepsilon>0$, then there is no solution to the inequality

$$
0<|u \tau-v+\mu|<A B^{-w}
$$

in positive integers $u, v$, and $w$ with

$$
u \leq M \quad \text { and } \quad w \geq \frac{\log (A q / \varepsilon)}{\log B} .
$$

At various occasions, we need to find a lower bound for linear forms in logarithms with bounded integer coefficients in three and four variables. In this case we use the LLL algorithm that we describe below. Let $\tau_{1}, \tau_{2}, \ldots \tau_{t} \in \mathbb{R}$ and the linear form

$$
x_{1} \tau_{1}+x_{2} \tau_{2}+\cdots+x_{t} \tau_{t} \quad \text { with } \quad\left|x_{i}\right| \leq X_{i}
$$

We put $X:=\max \left\{X_{i}\right\}, C>(t X)^{t}$ and consider the integer lattice $\Omega$ generated by

$$
\mathbf{b}_{j}:=\mathbf{e}_{j}+\left\lfloor C \tau_{j}\right\rceil \quad \text { for } \quad 1 \leq j \leq t-1 \quad \text { and } \quad \mathbf{b}_{t}:=\left\lfloor C \tau_{t}\right\rceil \mathbf{e}_{t}
$$

where $C$ is a sufficiently large positive constant.

Lemma 3. Let $X_{1}, X_{2}, \ldots, X_{t}$ be positive integers such that $X:=\max \left\{X_{i}\right\}$ and $C>$ $(t X)^{t}$ is a fixed sufficiently large constant. With the above notation on the lattice $\Omega$, we consider a reduced base $\left\{\boldsymbol{b}_{i}\right\}$ to $\Omega$ and its associated Gram-Schmidt orthogonalization base $\left\{\boldsymbol{b}_{i}^{*}\right\}$. We set

$$
c_{1}:=\max _{1 \leq i \leq t} \frac{\left\|\boldsymbol{b}_{1}\right\|}{\left\|\boldsymbol{b}_{i}^{*}\right\|}, \quad \theta:=\frac{\left\|\boldsymbol{b}_{1}\right\|}{c_{1}}, \quad Q:=\sum_{i=1}^{t-1} X_{i}^{2}, \quad \text { and } \quad R:=\frac{1}{2}\left(1+\sum_{i=1}^{t} X_{i}\right) .
$$


If the integers $x_{i}$ are such that $\left|x_{i}\right| \leq X_{i}$, for $1 \leq i \leq t$ and $\theta^{2} \geq Q+R^{2}$, then we have

$$
\left|\sum_{i=1}^{t} x_{i} \tau_{i}\right| \geq \frac{\sqrt{\theta^{2}-Q}-R}{C} .
$$

For the proof and further details, we refer the reader to the book of Cohen. (Proposition 2.3.20 in [5], pp. 58-63).

\section{BOUNDing THE VARIABLES}

We assume that $\left(x_{1}, y_{1}\right)$ is the smallest positive solution of the Pell equation (3). We set

and put

$$
x_{1}^{2}-d y_{1}^{2}=: \epsilon, \quad \epsilon \in\{ \pm 1\},
$$

$$
\delta:=x_{1}+\sqrt{d} y_{1} \quad \text { and } \quad \eta:=x_{1}-\sqrt{d} y_{1}=\epsilon \delta^{-1} .
$$

From (4), we get

$$
x_{n}=\frac{1}{2}\left(\delta^{n}+\eta^{n}\right) .
$$

Since $\delta \geq 1+\sqrt{2}=\alpha$, it follows that the estimate

$$
\frac{\delta^{n}}{\alpha} \leq x_{n}<\delta^{n}
$$

holds for all $n \geq 1$. The inequality on the right-hand side of $(13)$ follows because $|\eta|=|\delta|^{-1}<\delta$. For the inequality of the left-hand side of $(13)$, we note that

$$
x_{n} \geq \frac{\delta^{n}-\delta^{-n}}{2}=\delta^{n}\left(\frac{1-\delta^{-2 n}}{2}\right) \geq \delta^{n}\left(\frac{1-(1+\sqrt{2})^{-2}}{2}\right) \geq \frac{\delta^{n}}{1+\sqrt{2}}=\frac{\delta^{n}}{\alpha} .
$$

We let $(n, \ell, m):=\left(n_{i}, \ell_{i}, m_{i}\right)$ for $i=1,2$ be the solutions of (6). By (2) and (13), we get

$$
\alpha^{\ell+m-4} \leq P_{\ell} P_{m}=x_{n}<\delta^{n} \quad \text { and } \quad \frac{\delta^{n}}{\alpha} \leq x_{n}=P_{\ell} P_{m} \leq \alpha^{\ell+m-2}
$$

so

$$
n c_{1} \log \delta+1<\ell+m<n c_{1} \log \delta+4 \quad \text { where } \quad c_{1}:=\frac{1}{\log \alpha} .
$$

To fix ideas, we assume that

$$
m \geq \ell \quad \text { and } \quad n_{1}<n_{2} .
$$

We also put

$$
\ell_{3}:=\min \left\{\ell_{1}, \ell_{2}\right\}, \quad \ell_{4}:=\max \left\{\ell_{1}, \ell_{2}\right\}, \quad m_{3}:=\min \left\{m_{1}, m_{2}\right\}, \quad m_{4}:=\max \left\{m_{1}, m_{2}\right\} .
$$

Using the inequality (15) together with the fact that $\delta \geq 1+\sqrt{2}=\alpha\left(\right.$ so, $\left.c_{1} \log \delta>1\right)$, gives us that

$$
n_{2}<n_{2} c_{1} \log \delta<n_{2} c_{1} \log \delta+1<\ell_{2}+m_{2} \leq 2 m_{2} \leq 2 m_{4},
$$

so

$$
n_{1}<n_{2}<2 m_{4} .
$$


Thus, it is enough to find an upper bound on $m_{4}$. Substituting (1) and (12) in (6) we get

$$
\frac{1}{2}\left(\delta^{n}+\eta^{n}\right)=\frac{1}{8}\left(\alpha^{\ell}-\beta^{\ell}\right)\left(\alpha^{m}-\beta^{m}\right) .
$$

This can be regrouped as

$$
\delta^{n}\left(2^{2}\right) \alpha^{-\ell-m}-1=-4 \eta^{n} \alpha^{-\ell-m}-\left(\beta \alpha^{-1}\right)^{\ell}-\left(\beta \alpha^{-1}\right)^{m}+\left(\beta \alpha^{-1}\right)^{\ell+m} .
$$

Since $\beta=-\alpha^{-1}, \eta=\varepsilon \delta^{-1}$ and using the fact that $\delta^{n} \geq \alpha^{\ell+m-4}$ (by (14)), we get

$$
\begin{aligned}
\left|\delta^{n}\left(2^{2}\right) \alpha^{-\ell-m}-1\right| & \leq \frac{4}{\delta^{n} \alpha^{\ell+m}}+\frac{1}{\alpha^{2 \ell}}+\frac{1}{\alpha^{2 m}}+\frac{1}{\alpha^{2(\ell+m)}} \\
& \leq \frac{4 \alpha^{4}}{\alpha^{2(\ell+m)}}+\frac{3}{\alpha^{2 \ell}}<\frac{140}{\alpha^{2 \ell}}
\end{aligned}
$$

In the above, we have also used the facts that $m \geq \ell$ and $4 \alpha^{4}+3<140$. Hence,

$$
\left|\delta^{n}\left(2^{2}\right) \alpha^{-\ell-m}-1\right|<\frac{140}{\alpha^{2 \ell}}
$$

We let $\Lambda:=\delta^{n}\left(2^{2}\right) \alpha^{-\ell-m}-1$. We put

$$
\Gamma:=n \log \delta-2 \log 2-(\ell+m) \log \alpha .
$$

Note that $e^{\Gamma}-1=\Lambda$. If $\ell>100$, then $\frac{140}{\alpha^{2 \ell}}<\frac{1}{2}$. Since $\left|e^{\Gamma}-1\right|<1 / 2$, it follows that

$$
|\Gamma|<2\left|e^{\Gamma}-1\right|<\frac{280}{\alpha^{2 \ell}}
$$

By recalling that $(n, \ell, m)=\left(n_{i}, \ell_{i}, m_{i}\right)$ for $i=1,2$, we get that

$$
\left|n_{i} \log \delta-2 \log 2-\left(\ell_{i}+m_{i}\right) \log \alpha\right|<\frac{280}{\alpha^{2 \ell_{i}}}
$$

holds for both $i=1,2$ provided $\ell_{3}>100$.

We apply Theorem 2 on the left-hand side of (18). First, we need to check that $\Lambda \neq 0$. Well, if it were, then $\delta^{n} \alpha^{-\ell-m}=\frac{1}{4}$. However, this is impossible since $\delta^{n} \alpha^{-\ell-m}$ is a unit while $1 / 4$ is not. Thus, $\Lambda \neq 0$, and we can apply Theorem 2. We take the data

$$
t:=3, \quad \gamma_{1}:=\delta, \quad \gamma_{2}:=2, \quad \gamma_{3}:=\alpha, \quad b_{1}:=n, \quad b_{2}:=2, \quad b_{3}:=-\ell-m .
$$

We take $\mathbb{K}:=\mathbb{Q}(\sqrt{d}, \alpha)$ which has degree $D \leq 4$ (it could be that $d=2$ in which case $D=2$; otherwise, $D=4$ ). Since $\delta \geq 1+\sqrt{2}=\alpha$, the second inequality in (14) tells us that $n \leq \ell+m$, so we take $B:=2 m$. We have $h\left(\gamma_{1}\right)=h(\delta)=\frac{1}{2} \log \delta$, $h\left(\gamma_{2}\right)=h(2)=\log 2$ and $h\left(\gamma_{3}\right)=h(\alpha)=\frac{1}{2} \log \alpha$. Thus, we can take $A_{1}:=2 \log \delta$, $A_{2}:=4 \log 2$, and $A_{3}:=2 \log \alpha$. Now, Theorem 2 tells us that

$$
\begin{aligned}
\log |\Lambda| & >-1.4 \times 30^{6} \times 3^{4.5} \times 4^{2}(1+\log 4)(1+\log (2 m))(2 \log \delta)(4 \log 2)(2 \log \alpha) \\
& >-5.34 \times 10^{13} \log \delta(1+\log (2 m)) .
\end{aligned}
$$

By comparing the above inequality with $(18)$, we get

$$
2 \ell \log \alpha-\log 140<5.34 \times 10^{13} \log \delta(1+\log (2 m)) .
$$


Thus

$$
\ell<5.36 \times 10^{13} \log \delta(1+\log (2 m)) .
$$

Since, $\delta^{n}<\alpha^{\ell+m}$, we get that

$$
n \log \delta<(\ell+m) \log \alpha \leq 2 m \log \alpha,
$$

which together with the estimate 22 gives

$$
n \ell<5.35 \times 10^{13} m(1+\log (2 m)) .
$$

Let us record what we have proved, since this will be important later-on.

Lemma 4. If $x_{n}=P_{\ell} P_{m}$ and $m \geq \ell$, then

$\ell<5.36 \times 10^{13} \log \delta(1+\log (2 m)), n \ell<5.35 \times 10^{13} m(1+\log (2 m)), n \log \delta<2 m \log \alpha$.

Note that we did not assume that $\ell_{3}>100$ for Lemma 4 since we have worked with the inequality (18) and not with $(20)$. We now again assume that $\ell_{3}>100$. Then the two inequalities (21) hold. We eliminate the term involving $\log \delta$ by multiplying the inequality for $i=1$ with $n_{2}$ and the one for $i=2$ with $n_{1}$, subtract them and apply the triangle inequality as follows

$$
\begin{aligned}
& \left|2\left(n_{2}-n_{1}\right) \log 2-\left(n_{2}\left(\ell_{1}+m_{1}\right)-n_{1}\left(\ell_{2}+m_{2}\right)\right) \log \alpha\right| \\
& =\left|n_{2}\left(n_{1} \log \delta+2 \log 2-\left(\ell_{1}+m_{1}\right) \log \alpha\right)-n_{1}\left(n_{2} \log \delta+2 \log 2-\left(\ell_{2}+m_{2}\right) \log \alpha\right)\right| \\
& \leq n_{2}\left|n_{1} \log \delta+2 \log 2-\left(\ell_{1}+m_{1}\right) \log \alpha\right|+n_{1}\left|n_{2} \log \delta+2 \log 2-\left(\ell_{2}+m_{2}\right) \log \alpha\right| \\
& \leq \frac{280 n_{2}}{\alpha^{2 \ell_{1}}}+\frac{280 n_{1}}{\alpha^{2 \ell_{2}}}<\frac{560 n_{2}}{\alpha^{2 \ell_{3}}} .
\end{aligned}
$$

Thus,

$$
|\Gamma|:=\left|\left(n_{2}-n_{1}\right) \log 4-\left(n_{2}\left(\ell_{1}+m_{1}\right)-n_{1}\left(\ell_{2}+m_{2}\right)\right) \log \alpha\right|<\frac{560 n_{2}}{\alpha^{2 \ell_{3}}} .
$$

We are now set to apply Theorem 3 with the data

$$
t:=2, \quad \gamma_{1}:=4, \quad \gamma_{2}:=\alpha, \quad b_{1}:=n_{2}-n_{1}, \quad b_{2}:=n_{2}\left(\ell_{1}+m_{1}\right)-n_{1}\left(\ell_{2}+m_{2}\right) .
$$

The fact that $\gamma_{1}=2$ and $\gamma_{2}=\alpha$ are multiplicatively independent follows because $\alpha$ is a unit while 2 is not. We observe that $n_{2}-n_{1}<n_{2}$, whereas by the absolute value of the inequality in $(23)$, we have

$$
\left|n_{2}\left(\ell_{1}+m_{1}\right)-n_{1}\left(\ell_{2}+m_{2}\right)\right| \leq\left(n_{2}-n_{1}\right) \frac{2 \log 2}{\log \alpha}+\frac{560 n_{2}}{\alpha^{2 \ell_{3}} \log \alpha}<2 n_{2},
$$

because $\ell_{3}>100$. We have that $\mathbb{K}:=\mathbb{Q}(\alpha)$, which has $D:=2$. So we can take

$$
\log B_{1}=\max \left\{h\left(\gamma_{1}\right), \frac{\left|\log \gamma_{1}\right|}{2}, \frac{1}{2}\right\}=2 \log 2,
$$

and

$$
\log B_{2}=\max \left\{h\left(\gamma_{2}\right), \frac{\left|\log \gamma_{2}\right|}{2}, \frac{1}{2}\right\}=\frac{1}{2}
$$


Thus,

$$
b^{\prime}=\frac{\left|n_{2}-n_{1}\right|}{2 \log B_{2}}+\frac{\left|n_{2}\left(\ell_{1}+m_{1}\right)-n_{1}\left(\ell_{2}+m_{2}\right)\right|}{2 \log B_{1}} \leq n_{2}+\frac{n_{2}}{2 \log 2}<2 n_{2} .
$$

Now Theorem 3 tells us that with

$$
\Gamma=2\left(n_{2}-n_{1}\right) \log 2-\left(n_{2}\left(\ell_{1}+m_{1}\right)-n_{1}\left(\ell_{2}+m_{2}\right)\right) \log \alpha,
$$

we have

$$
\log |\Gamma|>-24.34 \times 2^{4}\left(\max \left\{\log \left(2 n_{2}\right)+0.14,10.5\right\}\right)^{2} \cdot(2 \log 2) \cdot(1 / 2) .
$$

Thus,

$$
\log |\Gamma|>-270\left(\max \left\{\log \left(2 n_{2}\right)+0.14,10.5\right\}\right)^{2} .
$$

By comparing the above inequality with (23), we get

$$
2 \ell_{3} \log \alpha-\log \left(240 n_{2}\right)<270\left(\max \left\{\log \left(2 n_{2}\right)+0.14,10.5\right\}\right)^{2} .
$$

If $n_{2} \leq 15785$, then $\log \left(2 n_{2}\right)+0.14<10.5$. Thus, the last inequality above gives

$$
2 \ell_{3} \log \alpha<270 \times 10.5^{2}+\log (240 \times 15785),
$$

giving $\ell_{3}<16000$ in this case. Otherwise, $n_{2}>15785$, and we get

$$
2 \ell_{3} \log \alpha<270\left(1+\log n_{2}\right)^{2}+\log \left(240 n_{2}\right)<280\left(1+\log n_{2}\right)^{2},
$$

which gives

$$
\ell_{3}<160\left(1+\log n_{2}\right)^{2} .
$$

We record what we have proved

Lemma 5. If $\ell_{3}>100$, then either

(i) $n_{2} \leq 15785$ and $\ell_{3}<16000$ or

(ii) $n_{2}>15785$, in which case $\ell_{3}<160\left(1+\log n_{2}\right)^{2}$.

Now suppose that some $\ell$ is fixed in (6), or at least we have some good upper bounds on it. We rewrite (6) using (1) and (12) as

$$
\frac{1}{2}\left(\delta^{n}+\eta^{n}\right)=\frac{P_{\ell}}{2 \sqrt{2}}\left(\alpha^{m}-\beta^{m}\right)
$$

SO

$$
\delta^{n}\left(\frac{\sqrt{2}}{P_{\ell}}\right) \alpha^{-m}-1=-\frac{\sqrt{2}}{P_{\ell}} \eta^{n} \alpha^{-m}-\left(\beta \alpha^{-1}\right)^{m} .
$$

Since $\ell \geq 1, \beta=-\alpha^{-1}, \eta=\varepsilon \delta^{-1}$, and $\delta^{n}>\alpha^{\ell+m-3}$, we get

$$
\begin{aligned}
\left|\delta^{n}\left(\frac{\sqrt{2}}{P_{\ell}}\right) \alpha^{-m}-1\right| & \leq \frac{\sqrt{2}}{P_{\ell} \delta^{n} \alpha^{m}}+\frac{1}{\alpha^{2 m}} \leq \frac{\sqrt{2} \alpha^{4}}{\alpha^{2(\ell+m)}}+\frac{1}{\alpha^{2 m}} \\
& \leq \frac{\sqrt{2} \alpha^{4}+1}{\alpha^{2 m}}<\frac{50}{\alpha^{2 m}},
\end{aligned}
$$


where we have used the fact that $m \geq \ell \geq 1$ and $\sqrt{2} \alpha^{4}+1<50$. Hence,

$$
\left|\Lambda_{1}\right|:=\left|\delta^{n}\left(\frac{\sqrt{2}}{P_{\ell}}\right) \alpha^{-m}-1\right|<\frac{50}{\alpha^{2 m}} .
$$

We assume that $m_{3}>100$. In particular, $\frac{50}{\alpha^{2 m}}<\frac{1}{2}$ for $m \in\left\{m_{1}, m_{2}\right\}$, so we get by the previous argument that

$$
\left|\Gamma_{1}\right|:=\left|n \log \delta+\log \left(\sqrt{2} / P_{\ell}\right)-m \log \alpha\right|<\frac{100}{\alpha^{2 m}} .
$$

We are now set to apply Theorem 2 on the left-hand side of (24) with the data

$$
t:=3, \quad \gamma_{1}:=\delta, \quad \gamma_{2}:=\sqrt{2} / P_{\ell}, \quad \gamma_{3}:=\alpha, \quad b_{1}:=n, \quad b_{2}:=1, \quad b_{3}:=-m .
$$

First, we need to check that $\Lambda_{1}:=\delta^{n}\left(\sqrt{2} / P_{\ell}\right) \alpha^{-m}-1 \neq 0$. If not, then $\delta^{n}=\alpha^{m} P_{\ell} / \sqrt{2}$. The left-hand side belongs to the field $\mathbb{Q}(\sqrt{d})$ but not rational while the right-hand side belongs to the field $\mathbb{Q}(\sqrt{2})$. This is not possible unless $d=2$. In this last case, $\delta$ is a unit in $\mathbb{Q}(\sqrt{2})$ while $P_{\ell} / \sqrt{2}$ is not a unit in $\mathbb{Q}(\sqrt{2})$ since the norm of this last element is $P_{\ell}^{2} / 2 \neq \pm 1$. So $\Lambda_{1} \neq 0$. Thus, we can apply Theorem 2 . We have the field $\mathbb{K}:=\mathbb{Q}(\sqrt{d}, \sqrt{2})$ which has degree $D \leq 4$. We also have

$$
h\left(\gamma_{1}\right)=\frac{1}{2} \log \delta, \quad h\left(\gamma_{2}\right)=\max \left\{\frac{1}{2} \log 2, \log P_{\ell}\right\}, \quad \text { and } \quad h\left(\gamma_{3}\right)=\frac{1}{2} \log \alpha .
$$

Since $P_{\ell} \leq \alpha^{\ell-1}<2^{2 \ell}$, we can take

$$
A_{1}:=2 \log \delta, \quad A_{2}:=8 \ell \log 2, \quad \text { and } \quad A_{3}:=2 \log \alpha .
$$

Then, by Theorem 2 we get

$$
\begin{aligned}
\log \left|\Lambda_{1}\right| & >-1.4 \times 30^{6} \times 3^{4.5} \times 4^{2}(1+\log 4)(1+\log m)(2 \log \delta)(8 \ell \log 2)(2 \log \alpha) \\
& >-7.58 \times 10^{13} \ell \log \delta(1+\log m) .
\end{aligned}
$$

Comparing the above inequality with 24 , we get

$$
2 m \log \alpha-\log 50<7.58 \times 10^{13} \ell \log \delta(1+\log m),
$$

which implies that

$$
m<4.30 \times 10^{13} \ell \log \delta(1+\log m) .
$$

We record what we have proved.

Lemma 6. If $x_{n}=P_{\ell} P_{m}$ with $m \geq \ell \geq 1$, then we have

$$
m<4.30 \times 10^{13} \ell \log \delta(1+\log m) .
$$

Note that we did not use the assumption that $\ell_{3}>100$ of that $m_{3}>100$ for Lemma 6 since we worked with the inequality (24) not with the inequality (25). We now assume 
that $m_{3}>100$ and in particular (25) holds for $(n, \ell, m)=\left(n_{i}, \ell_{i}, m_{i}\right)$ for both $i=1,2$. By the previous procedure, we also eliminate the term involving $\log \delta$ as follows

$$
\begin{aligned}
\left|n_{2} \log \left(\sqrt{2} / P_{\ell_{1}}\right)-n_{1} \log \left(\sqrt{2} / P_{\ell_{2}}\right)-\left(n_{2} m_{1}-n_{1} m_{2}\right) \log \alpha\right| & <\frac{100 n_{2}}{\alpha^{2 m_{1}}}+\frac{100 n_{1}}{\alpha^{2 m_{2}}} \\
& <\frac{200 n_{2}}{\alpha^{2 m_{3}}} .
\end{aligned}
$$

We assume that $\alpha^{2 m_{3}}>400 n_{2}$. If we put

$$
\Gamma_{2}:=n_{2} \log \left(\sqrt{2} / P_{\ell_{1}}\right)-n_{1} \log \left(\sqrt{2} / P_{\ell_{2}}\right)-\left(n_{2} m_{1}-n_{1} m_{2}\right) \log \alpha
$$

we have that $\left|\Gamma_{2}\right|<1 / 2$. We then get that

$$
\left|\Lambda_{2}\right|:=\left|e^{\Gamma_{2}}-1\right|<2\left|\Gamma_{2}\right|<\frac{400 n_{2}}{\alpha^{2 m_{3}}} .
$$

We apply Theorem 2 to

$$
\Lambda_{2}:=\left(\sqrt{2} / P_{\ell_{1}}\right)^{n_{2}}\left(\sqrt{2} / P_{\ell_{2}}\right)^{-n_{1}} \alpha^{-\left(n_{2} m_{1}-n_{1} m_{2}\right)}-1 .
$$

First, we need to check that $\Lambda_{2} \neq 0$. Well, if it were, then it would follow that

$$
\frac{P_{\ell_{2}}^{n_{1}}}{P_{\ell_{1}}^{n_{2}}}=2^{\left(n_{1}-n_{2}\right) / 2} \alpha^{n_{2} m_{1}-n_{1} m_{2}} .
$$

By squaring the above relation, we get that $\alpha^{2\left(n_{2} m_{1}-n_{1} m_{2}\right)} \in \mathbb{Q}$, so $n_{2} m_{1}=m_{2} n_{1}$. Thus, $P_{\ell_{2}}^{n_{1}} / P_{\ell_{1}}^{n_{2}}=2^{\left(n_{1}-n_{2}\right) / 2}$. If $n_{1}=n_{2}$, then together with $n_{2} m_{1}=n_{2} m_{2}$ we get $m_{1}=m_{2}$ and now from $x_{n_{i}}=P_{\ell_{i}} P_{m_{i}}$, we get that $P_{\ell_{1}}=P_{\ell_{2}}$, so $\ell_{1}=\ell_{2}$. This is impossible. If $\ell_{4} \geq 2$ then the Carmichael Primitive Divisor Theorem for Pell numbers says that if $\ell_{3} \neq \ell_{4}\left(\right.$ so $\left.\ell_{1} \neq \ell_{2}\right)$, then $P_{\ell_{4}}$ has a multiple of a prime $\geq 2$ which does not divide $P_{\ell_{3}}$. This is not possible in our case. So, still under the assumption that $\ell_{4} \geq 2$, we get that $\ell_{1}=\ell_{2}$ so $P_{\ell_{1}}^{n_{1}-n_{2}}=2^{\left(n-n_{1}\right) / 2}$, giving that $P_{\ell}=\sqrt{2}$, a contradiction. Thus, $\ell_{4} \leq 2$. Also the previous argument shows that $\ell_{1} \neq \ell_{2}$. We now list all the Pell numbers with indices at most 2. The only ones which is a multiple of 2 is $P_{2}=2$. So $2 \in\left\{\ell_{1}, \ell_{2}\right\}$. It follows that the other index has to be 1 since the only indices $k<2$ such that $P_{k}$ is a power of 2 . Since $n_{1}<n_{2}$, the exponent $\left(n_{1}-n_{2}\right) / 2$ of 2 is negative, so it follows that $\ell_{1}=2$ and $\ell_{2}=1$. So we get the equation $2^{-n_{2}}=2^{\left(n_{1}-n_{2}\right) / 2}$, which does not yield positive integer solutuions in $n_{1}, n_{2}$. So $\Lambda_{2} \neq 0$. Thus, we can now apply Theorem 2 with the data

$$
\begin{array}{cl}
t:=3, \quad \gamma_{1}:=\sqrt{5} / P_{\ell_{1}}, & \gamma_{2}:=\sqrt{5} / P_{\ell_{1}}, \quad \gamma_{3}:=\alpha, \quad b_{1}=n_{2}, \\
b_{2}:=-n_{1}, \quad b_{3}:=-\left(n_{2} m_{1}-n_{1} m_{2}\right) .
\end{array}
$$

We have $\mathbb{K}:=Q(\sqrt{2})$ which has degree $D:=2$. Also, using $(16)$, we can take $B:=2 m_{4}^{2}$. We can also take $A_{1}:=4 \ell_{1} \log 2, A_{2}:=4 \ell_{2} \log 2$, and $A_{3}:=\log \alpha$. Theorem 2 gives that

$$
\begin{aligned}
\log \left|\Lambda_{2}\right| & >-1.4 \times 30^{6} \times 3^{4.5} \times 2^{2}(1+\log 2)\left(1+\log \left(2 m_{4}^{2}\right)\right)\left(4 l_{1} \log 2\right)\left(4 l_{2} \log 2\right) \log \alpha \\
& >-6.57 \times 10^{12} \ell_{1} \ell_{2}\left(1+\log \left(2 m_{4}^{2}\right)\right) .
\end{aligned}
$$


By comparing this with the inequality (27), we get

$$
2 m_{3} \log \alpha-\log \left(400 n_{2}\right)<6.57 \times 10^{12} \ell_{1} \ell_{2}\left(1+\log \left(2 m_{4}^{2}\right)\right) .
$$

Since $n_{2}<2 m_{4}$ and $m_{4}>100$, we get that $\log \left(48 n_{2}\right)<1+\log \left(2 m_{4}^{2}\right)$. Thus,

$$
m_{3}<6.6 \times 10^{12} \ell_{1} \ell_{2}\left(1+\log \left(2 m_{4}^{2}\right)\right) .
$$

All this was done under the assumption that $\alpha^{2 m_{3}}>400 n_{2}$. But if that inequality fails, then

$$
m_{3}<c_{1} \log \left(400 n_{2}\right)<12\left(1+\log \left(2 m_{4}^{2}\right)\right),
$$

which is much better than (28). Thus, (28) holds in all cases. Next, we record what we have proved.

Lemma 7. Assume that $m_{3}>100$, then we have

$$
m_{3}<6.6 \times 10^{12} \ell_{1} \ell_{2}\left(1+\log \left(2 m_{4}^{2}\right)\right) .
$$

We now start finding effective bounds for our variables.

Case 1. $\ell_{4} \leq 100$.

Then $\ell_{1}<100$ and $\ell_{2}<100$. By Lemma 7, we get that

$$
m_{3}<6.6 \times 10^{16}\left(1+\log \left(2 m_{4}^{2}\right)\right) .
$$

By Lemma 4, we get

$$
\log \delta<2 m_{3} \log \alpha<6.6 \times 10^{16}\left(1+\log \left(2 m_{4}^{2}\right)\right) .
$$

By the inequality (15), we have that

$$
\begin{aligned}
m_{4} & \leq m_{4}+\ell_{4}-1 \\
& <n_{2} c_{1} \log \delta+2 \\
& <\frac{1}{\log \alpha}\left(5.36 \times 10^{13}\left(1+\log \left(2 m_{4}\right)\right)\right)\left(6.6 \times 10^{16}\left(1+\log \left(2 m_{4}^{2}\right)\right)\right) \\
& <4 \times 10^{30} \log \left(1+\log \left(2 m_{4}\right)\right)\left(1+\log \left(2 m_{4}^{2}\right)\right) .
\end{aligned}
$$

With the help of Mathematica, we get that $m_{4}<5.3 \times 10^{34}$. Thus, using (16), we get

$$
\max \left\{n_{2}, m_{4}\right\}<1.1 \times 10^{35} .
$$

We record what we have proved.

Lemma 8. If $\ell_{4}:=\max \left\{\ell_{1}, \ell_{2}\right\} \leq 100$, then

$$
\max \left\{n_{2}, m_{4}\right\}<1.1 \times 10^{35} .
$$

From now on, we assume that $\ell_{4}>100$. Note that either $\ell_{3} \leq 100$ or $\ell_{3}>100$ case in which by Lemma 5 and the inequality 16 , we have $\ell_{3} \leq 160\left(1+\log \left(2 m_{4}\right)\right)^{2}$ provided that $m_{4}>10000$, which we now assume. 
We let $i \in\{1,2\}$ be such that $\ell_{i}=\ell_{3}$ and $j$ be such that $\{i, j\}=\{1,2\}$. We assume that $m_{3}>100$. We work with (25) for $i$ and (21) for $j$ and noting the conditions $m_{i}>100$ and $\ell_{j}=\ell_{4}>100$ are fullfilled. That is,

$$
\begin{gathered}
\left|n_{i} \log \delta+\log \left(\sqrt{2} / P_{\ell_{i}}\right)-m_{i} \log \alpha\right|<\frac{100}{\alpha^{2 m_{i}}}, \\
\left|n_{j} \log \delta-2 \log 2-\left(\ell_{j}+m_{j}\right) \log \alpha\right|<\frac{280}{\alpha^{2 \ell_{j}}} .
\end{gathered}
$$

By a similar procedure as before, we eliminate the term involving $\log \delta$. We multiply the first inequality by $n_{j}$, the second inequality by $n_{i}$, subtract the resulting inequalities and apply the triangle inequalty to get

$$
\begin{aligned}
\left|n_{j} \log \left(\sqrt{2} / P_{\ell_{i}}\right)-2 n_{i} \log 2-\left(n_{j} m_{i}-n_{i}\left(m_{j}+\ell_{j}\right)\right) \log \alpha\right| & <\frac{100 n_{j}}{\alpha^{2 m_{i}}}+\frac{280 n_{i}}{\alpha^{2 l_{j}}} \\
& <\frac{380 n_{2}}{\alpha^{2 \min \left\{m_{i}, \ell_{j}\right\}}} .
\end{aligned}
$$

Assume that $\alpha^{2 \min \left\{m_{i}, \ell_{j}\right\}}>760 n_{2}$. We put

$$
\Gamma_{3}:=n_{j} \log \left(\sqrt{2} / P_{\ell_{i}}\right)-2 n_{i} \log 2-\left(n_{j} m_{i}-n_{i}\left(m_{j}+\ell_{j}\right)\right) \log \alpha .
$$

We can write $\Lambda_{3}:=\left(\sqrt{2} / P_{\ell_{i}}\right)^{n_{j}} 2^{-2 n_{i}} \alpha^{-\left(n_{j} m_{i}+n_{i}\left(m_{j}+\ell_{j}\right)\right)}-1$. Under the above assumption and using (29), we get that

$$
\left|\Lambda_{3}\right|=\left|e^{\Gamma_{3}}-1\right|<2\left|\Gamma_{3}\right|<\frac{760 n_{2}}{\alpha^{2 \min \left\{m_{i}, \ell_{j}\right\}}} .
$$

We are now set to apply Theorem 2 on $\Lambda_{3}$. First, we need to check that $\Lambda_{3} \neq 0$. Well, if it were, then we would get that

$$
P_{\ell_{i}}^{n_{j}}=2^{-2 n_{i}+n_{j} / 2} \alpha^{n_{j} m_{i}-n_{i}\left(m_{j}+\ell_{j}\right)} .
$$

We study particular cases of 31. If $\ell_{i}=1$, then we have that

$$
2^{n_{j} / 2-2 n_{i}}=\alpha^{n_{j} m_{i}-n_{i}\left(m_{j}+\ell_{j}\right)},
$$

which holds when $n_{j}=4 n_{i}$ and $n_{j} m_{i}=n_{i}\left(m_{j}+\ell_{j}\right)$. Since $n_{j}=4 n_{i}$, it follows that $j=2, i=1$. Now substituting this in $n_{j} m_{i}=n_{i}\left(m_{j}+\ell_{j}\right)$, we get $\left(4 n_{1}\right) m_{1}=n_{1}\left(m_{2}+\ell_{2}\right)$, so $m_{2}=4 m_{1}-\ell_{2}$. Thus, we have

$$
x_{n_{1}}=P_{\ell_{1}} P_{m_{1}}=P_{m_{1}}, \quad x_{n_{2}}=x_{4 n_{1}}=P_{\ell_{2}} P_{m_{2}}=P_{\ell_{2}} P_{4 m_{1}-\ell_{2}} .
$$

Since $x_{4 n_{1}}=2\left(2 x_{n_{1}}^{2} \pm 1\right)^{2}-1$, we get that

$$
2\left(2 P_{m_{1}}^{2} \pm 1\right)^{2}-1=P_{\ell_{2}} P_{4 m_{1}-\ell_{2}} .
$$

If $\ell_{i}=2$, then we get that

$$
2^{n_{j} / 2+2 n_{i}}=\alpha^{n_{j} m_{i}-n_{i}\left(m_{j}+\ell_{j}\right)},
$$

which holds when $n_{j}+4 n_{i}=0$ and $n_{j} m_{i}=n_{i}\left(m_{j}+\ell_{j}\right)$. This is false for $n_{i}, n_{j}>0$. 
For $\ell_{i}>2$, Carmichael Primitive Divisor Theorem for Pell numbers, we get a contradiction on (31). Thus, we can apply Theorem 2 with the data

$$
\begin{gathered}
t:=3, \quad \gamma_{1}:=\sqrt{2} / P_{\ell_{i}}, \quad \gamma_{2}:=2 \quad \gamma_{3}:=\alpha \quad b_{1}:=n_{j}, \\
b_{2}:=-2 n_{i}, \quad b_{3}:=-\left(n_{j} m_{i}-n_{i}\left(m_{j}+\ell_{j}\right)\right) .
\end{gathered}
$$

From the previous calculations, we know that $\mathbb{K}:=\mathbb{Q}(\sqrt{2})$ which has degree $D:=2$ and $A_{1}:=4 \ell_{i} \log 2, A_{2}:=2 \log 2$, and $A_{3}:=2 \log \alpha$. We also take $B:=2 m_{4}^{2}$. By Theorem 2, we get that

$$
\begin{aligned}
\log \left|\Lambda_{3}\right| & >-1.4 \times 30^{6} \times 3^{4.5} \times 2^{2}(1+\log 2)\left(1+\log \left(2 m_{4}^{2}\right)\right)\left(4 \ell_{i} \log 2\right)(2 \log 2) \log \alpha, \\
& >-3.30 \times 10^{12} \ell_{i}\left(1+\log \left(2 m_{4}^{2}\right)\right) .
\end{aligned}
$$

Comparing the above inequality with (30), we get

$$
2 \min \left\{m_{i}, \ell_{j}\right\} \log \alpha-\log \left(760 n_{2}\right)<3.30 \times 10^{12} l_{i}\left(1+\log \left(2 m_{4}\right)\right) .
$$

Since $m_{4}>100$, we get using $(16)$ that $n_{2}<2 m_{4}$. Hence,

$$
\min \left\{m_{i}, l_{j}\right\}<\frac{c_{1}}{2} 3.30 \times 10^{12} \times 160\left(1+\log \left(2 m_{4}\right)\right)^{2}\left(1+\log \left(2 m_{4}^{2}\right)\right)+\frac{c_{1}}{2} \log \left(880 m_{4}^{2}\right),
$$

which implies that

$$
\min \left\{m_{i}, \ell_{j}\right\}<3 \times 10^{15}\left(1+\log \left(2 m_{4}\right)\right)^{2}\left(1+\log \left(2 m_{4}^{2}\right)\right) .
$$

All this was under the assumptions that $m_{4}>10000$, and that $\alpha^{2 \min \left\{m_{i}, \ell_{j}\right\}}>440 n_{2}$. But, still under the condition that $m_{4}>10000$, if $\alpha^{2 \min \left\{m_{i}, \ell_{j}\right\}}<440 n_{2}$, then we get an inequality for $\min \left\{m_{i}, \ell_{j}\right\}$ which is even much better than 32 . So, (32) holds provided that $m_{4}>10000$. Suppose say that $\min \left\{m_{i}, \ell_{j}\right\}=\ell_{j}$. Then we get that

$$
\ell_{3}<160\left(1+\log \left(2 m_{4}\right)\right)^{2}, \quad \ell_{4}<3 \times 10^{15}\left(1+\log \left(2 m_{4}\right)\right)^{2}\left(1+\log \left(2 m_{4}^{2}\right)\right) .
$$

By Lemma 7, since $m_{3}>100$, we get

$$
\begin{aligned}
m_{3} & <\left(6.6 \times 10^{12}\right)\left(160\left(1+\log \left(2 m_{4}\right)\right)^{2}\right)\left(1+\log \left(2 m_{4}^{2}\right)\right) \\
& \times 3 \times 10^{15}\left(1+\log \left(2 m_{4}\right)\right)^{2}\left(1+\log \left(2 m_{4}^{2}\right)\right) \\
& <3.2 \times 10^{30}\left(1+\log \left(2 m_{4}^{2}\right)\right)^{6} .
\end{aligned}
$$

Together with Lemma 4, we get

$$
\log \delta<3.2 \times 10^{30}\left(1+\log \left(2 m_{4}^{2}\right)\right)^{6},
$$

which together with Lemma 6 gives

$$
\begin{aligned}
m_{4}< & 4.30 \times 10^{13}\left(3 \times 10^{15}\left(1+\log \left(2 m_{4}\right)\right)^{2}\left(1+\log \left(2 m_{4}^{2}\right)\right)\right) \\
& \times\left(3.2 \times 10^{30}\left(1+\log \left(2 m_{4}^{2}\right)\right)^{6}\right)\left(1+\log m_{4}\right),
\end{aligned}
$$

which implies that

$$
m_{4}<4.1 \times 10^{59}\left(1+\log \left(2 m_{4}^{2}\right)\right)^{10} .
$$


With the help of Mathematica we get that $m_{4}<3.8 \times 10^{85}$. This was proved under the assumption that $m_{4}>10000$, but the situation $m_{4} \leq 10000$ already provides a better bound than $m_{4}<3.8 \times 10^{85}$. Hence,

$$
\max \left\{n_{2}, m_{1}, m_{2}\right\}<3.8 \times 10^{85} .
$$

This was when $\ell_{j}=\min \left\{m_{i}, \ell_{j}\right\}$. Now we assume that $m_{i}=\min \left\{m_{i}, \ell_{j}\right\}$. Then we get

$$
m_{i}<3 \times 10^{15}\left(1+\log \left(2 m_{4}^{2}\right)\right)^{3} .
$$

By Lemma 4, we get that

$$
\log \delta<3 \times 10^{15}\left(1+\log \left(2 m_{4}^{2}\right)\right)^{3} .
$$

Now by Lemma 7 together with Lemma 4 to bound $l_{4}$ give

$$
\begin{aligned}
m_{4} & <4.30 \times 10^{13}\left(5.36 \times 10^{13}\left(3 \times 10^{15}\left(1+\log \left(2 m_{4}^{2}\right)\right)^{3}\right)(1+\log (2 m))\right)^{2} \\
& \left.\times\left(1+\log \left(2 m_{4}^{2}\right)\right)\right)\left(3 \times 10^{15}\left(1+\log \left(2 m_{4}^{2}\right)\right)^{3}\right)\left(1+\log m_{4}\right), \\
& <2 \times 10^{58}\left(1+\log \left(2 m_{4}^{2}\right)\right)^{10} .
\end{aligned}
$$

This gives, $m_{4}<1.6 \times 10^{84}$ which is a better bound than $3.8 \times 10^{85}$. We record what we have proved.

Lemma 9. If $\ell_{4}:=\max \left\{\ell_{1}, \ell_{2}\right\}>100$ and $m_{3}:=\min \left\{m_{1}, m_{2}\right\}>100$, then

$$
\max \left\{n_{2}, m_{1}, m_{2}\right\}<3.8 \times 10^{85} \text {. }
$$

It now remains the case when $\ell_{4}>100$ and $m_{3} \leq 100$. But then, by Lemma 4, we get $\log \delta<100$ and now Lemma 4 together with Lemma 7 give

$$
m_{4}<2 \times 10^{31}\left(1+\log \left(2 m_{4}^{2}\right)\right)^{3}
$$

which implies that $m_{4}<10^{38}$ and further $\max \left\{n_{1}, m_{1}, m_{2},\right\}<10^{40}$. We record what we have proved.

Lemma 10. If $\ell_{4}>100$ and $m_{3} \leq 100$, then

$$
\max \left\{n_{1}, m_{1}, m_{2},\right\}<10^{40} \text {. }
$$

\section{The FinAl COMPUtations}

We return to (23) and we set $s:=n_{2}-n_{1}$ and $r:=n_{2}\left(\ell_{1}+m_{1}\right)-n_{1}\left(\ell_{2}+m_{2}\right)$ and divide both sides by $s \log \alpha$ to get

$$
\left|\frac{\log 4}{\log \alpha}-\frac{r}{s}\right|<\frac{240 n_{2}}{\alpha^{2 \ell_{3}} s \log \alpha} .
$$

We assume that $\ell_{3}$ is so large that the right-hand side of the inequality in (34) is smaller than $1 /\left(2 s^{2}\right)$. This certainly holds if

$$
\alpha^{2 \ell_{3}}>480 n_{2}^{2} / \log \alpha
$$

Since $n_{2}<3.8 \times 10^{85}$, it follows that the last inequality (35) holds provided that $\ell_{3} \geq 227$, which we now assume. In this case $r / s$ is a convergent of the continued fraction of $\tau:=\log 4 / \log \alpha$ and $s<3.8 \times 10^{85}$. We are now set to apply Lemma 1 . 
We write $\tau:=\left[a_{0} ; a_{1}, a_{2}, a_{3}, \ldots\right]=[1,1,1,2,1,13,2,1,5,4,1,3,1,8,1,10,1,1,2,3, \ldots]$ for the continued fraction of $\tau$ and $p_{k} / q_{k}$ for the $k$-th convergent. We get that $r / s=$ $p_{j} / q_{j}$ for some $j \leq 170$. Furthermore, putting $a(M):=\max \left\{a_{j}: j=0,1, \ldots, 170\right\}$, we get $a(M):=1469$. By Lemma 1, we get

$$
\frac{1}{1471 s^{2}}=\frac{1}{(a(M)+2) s^{2}} \leq\left|\tau-\frac{r}{s}\right|<\frac{240 n_{2}}{\alpha^{2 l_{3}} s \log \alpha}
$$

giving

$$
\alpha^{2 \ell_{3}}<\frac{1471 \times 240 n_{2}^{2}}{\log \alpha}<\frac{1471 \times 240 \times\left(3.8 \times 10^{85}\right)^{2}}{\log \alpha},
$$

leading to $\ell_{3} \leq 230$. We record what we have just proved.

Lemma 11. We have $\ell_{3} \leq 230$.

If $\ell_{1}=\ell_{3}$, then we have $i=1$ and $j=2$, otherwise $\ell_{2}=\ell_{3}$ implying that we have $i=2$ and $j=1$. In both cases, the next step is the application of Lemma 3 (LLL algorithm) for (29), where $n_{i}<3.8 \times 10^{85}$ and $\left|n_{j} m_{i}-n_{i} m_{j}+n_{i} \ell_{j}\right|<10^{90}$. For each $\ell_{j} \in[1,230]$ and

$$
\Gamma_{3}:=n_{j} \log \left(\sqrt{2} / P_{\ell_{i}}\right)-2 n_{i} \log 2-\left(n_{j} m_{i}-n_{i} m_{j}+n_{i} \ell_{j}\right) \log \alpha
$$

we apply the LLL algorithm on $\Gamma_{3}$ with the data

$$
\begin{array}{r}
t:=3, \quad \tau_{1}:=\log \left(\sqrt{2} / P_{\ell_{i}}\right), \quad \tau_{2}:=\log 4, \quad \tau_{3}:=\log \alpha \\
x_{1}:=n_{j}, \quad x_{2}:=n_{i}, \quad x_{3}:=n_{j} m_{i}-n_{i} m_{j}+n_{i} \ell_{j} .
\end{array}
$$

Further, we set $X:=10^{90}$ as an upper bound to $\left|x_{i}\right|<2 n_{2}$ for $i=1,2$, and $C:=(5 X)^{5}$. A computer search in Mathematica allows us to conculde, together with the inequality 29 , that

$$
2 \times 10^{-220}<\min _{1 \leq \min \left\{m_{i}, l_{j}\right\} \leq 230}\left|\Gamma_{3}\right|<\frac{220 n_{2}}{\alpha^{2 \min \left\{m_{i}, l_{j}\right\}}} .
$$

Thus, $\min \left\{m_{i}, \ell_{j}\right\} \leq 401$.

We assume first that $i=1, j=2$. Thus, $\min \left\{m_{1}, \ell_{2}\right\} \leq 401$ can be split into two branches. If $m_{1} \leq 401$, then $\ell_{1}+m_{1} \leq 631$, and by (15) we obtain $n_{1}<556$. For $\ell_{2} \leq 401$ we run the LLL algorithm on (26) with $2 \leq \ell_{1} \leq 230$ and $\ell_{1} \leq \ell_{2} \leq 401$ for each $n_{i}<3.8 \times 10^{85}$ and further $\left|n_{2} m_{1}-n_{1} m_{2}\right|<10^{90}$. This results in the upper bound $m_{3} \leq 412$. This in turn splits into either $m_{1} \leq 412$ or $m_{2} \leq 412$. Suppose that $m_{1} \leq 412$, together with $\ell_{1} \leq 230$ and (15), it yields $n_{1} \leq 565$. For $m_{2} \leq 412$ and that $\ell_{2} \leq 401$, and then (15) gives $n_{2} \leq 716$. Clearly, now $n_{1} \leq 715$. The symmetric case $i=2, j=1$ with $\min \left\{m_{2}, \ell_{1}\right\} \leq 401$ is anologous. We record the results of the computation in the table below. 


\begin{tabular}{ccccccc}
\hline & $\ell_{1}$ & $m_{1}$ & $n_{1}$ & $\ell_{2}$ & $m_{2}$ & $n_{2}$ \\
\hline 1. & 230 & 401 & 556 & & & \\
2. & 230 & 412 & 565 & 401 & & \\
3. & 230 & & 715 & 401 & 412 & 716 \\
4. & & & 552 & 230 & 401 & 556 \\
5. & 401 & 412 & 716 & 230 & & \\
6. & 401 & & 556 & 230 & 412 & 565 \\
\hline
\end{tabular}

By similar arguments given in Kafle, et al. [10] by applying Lemma 1, Lemma 2 and Lemma 3 on the appropraite linear forms in logarithms, we can further reduce these bounds to

$$
\ell_{1} \leq 200, \quad m_{1} \leq 200, \quad \ell_{2} \leq 120, \quad m_{2} \leq 120, \quad n_{2} \leq 150
$$

The final verification of our results was carried out according to the bounds in (37) to check all the possibilities. With the help of a computer search in Mathematica we found no values of $d$ that lead to at least two positive integer solutions to (6). This completes the proof of Theorem 1 .

\section{ACKNOWLEDGEMENTS}

M. D. was supported by the Austrian Science Fund (FWF) grants: F5510-N26 Part of the special research program (SFB), "Quasi Monte Carlo Metods: Theory and Applications" and W1230 - "Doctoral Program Discrete Mathematics". F. L. was also supported by grant CPRR160325161141 from the NRF of South Africa, grant RTNUM19 from CoEMaSS, Wits, South Africa.

\section{REFERENCES}

[1] A. Baker and H. Davenport, The equations $3 x^{2}-2=y^{2}$ and $8 x^{2}-7=z^{2}$, Quart. J. Math. Oxford Ser. (2), 20 (1) (1969), 129-137.

[2] A. Baker and G. Wüstholz, Logarithmic forms and Diophantine geometry, vol. 9, Cambridge University Press, 2008.

[3] E. Bombieri and W. Gubler, Heights in Diophantine Geometry, Cambridge University Press, Cambridge, 2006.

[4] Y. Bugeaud, M. Mignotte, and S. Siksek, Classical Classical and modular approaches to exponential Diophantine equations I. Fibonacci and Lucas perfect powers, Ann. of Math. 163 (2) (2006), 969-1018.

[5] H. Cohen, Number Theory. Volume I: Tools and Diophantine Equations, Graduate Texts in Mathematics 239, Springer, 2007.

[6] M. Ddamulira, On the $x$-coordinates of Pell equations that are products of two Lucas numbers, Fibonacci Quart. 58 (1) (2020), 18-37.

[7] M. Ddamulira, F. Luca, and M. Rakotomalala, Fibonacci numbers which are products of two Pell numbers, Fibonacci Quart. 54 (1) (2016), 11-18.

[8] M. Ddamulira and F. Luca, On the $x$-coordinates of Pell equations which are $k$-generalized Fibonacci numbers, J. Number Theory 207 (2020), 156-195.

[9] A. Dujella and A. Pethö, A generalization of a theorem of Baker and Davenport, Quart. J. Math. Oxford Ser. (2) 49 (195) (1998), 291-306.

[10] B. Kafle, F. Luca, A. Montejano, L. Szalay, and A. Togbé, On the $x$-coordinates of Pell equations which are products of two Fibonacci numbers, J. Number Theory 203 (2019), 310-333. 
[11] B. Kafle, F. Luca, and A. Togbé, On the $x$-coordinates of Pell equations which are Fibonacci numbers II, Colloq. Math. 149 (1) (2017), 75-85.

[12] B. Kafle, F. Luca, and A. Togbé, $x$-Coordinates of Pell equations which are Tribonacci numbers II, Period. Math. Hungar. 79 (2) (2019), 157-167.

[13] B. Kafle, F. Luca, and A. Togbé, $X$-coordinates of Pell equations which are Lucas numbers, Bol. Soc. Mat. Mex. 25 (3) (2019), 481-493.

[14] M. Laurent, M. Mignotte, and Yu. Nesterenko, Formes linéaires en deux logarithmes et déterminants d'interpolation, J. Number Theory 55 (2) (1995), 285-321.

[15] F. Luca, A. Montejano, L. Szalay, and A. Togbé, On the $x$-coordinates of Pell equations which are Tribonacci numbers, Acta Arith. 179 (1) (2017), 25-35.

[16] F. Luca and A. Togbé, On the $x$-coordinates of Pell equations which are Fibonacci numbers, Math. Scand. 122 (1) (2018), 18-30.

[17] E. M. Matveev, An explicit lower bound for a homogeneous rational linear form in the logarithms of algebraic numbers II, Izv. Ross. Akad. Nauk Ser. Mat. 64 (6) (2000), 125-180, in Russian; English translation in Izv. Math. 64 (6) (2000), 1217-1269.

[18] S. S. Rihane, M. O. Hernane, and A. Togbé, The $x$-coordinates of Pell equations and Padovan numbers, Turkish J. Math. 43 (1) (2019), 207-223.

[19] OEIS Foundation Inc. (2019), The On-Line Encyclopedia of Integer Sequences, https://oeis. org/A000129.

Mahadi Ddamulira

Institute of ANALYSIS AND Number Theory

Graz University of TeChNOLOGY

KopernikUSGaSSE 24/II

A-8010 Graz, Austria

E-mail address: mddamulira@tugraz.at; mahadi@aims.edu.gh

FLORIAN LUCA

School of Mathematics, University of the WitwatersRand

Private BAG X3

WITS 2050

Johannesburg, South Africa

Research Group in Algebraic Structures and Applications, Jeddah, Saudi Arabia

Centro de Ciencias Matemáticas, unam, Morelia, Mexico

E-mail address: Florian.Luca@wits.ac.za 